\title{
Excited Charmed Baryons
}

\author{
Elena Solovieva ${ }^{1,2, \star}$ \\ ${ }^{1}$ Moscow Institute of Physics and Technology, 9 Institutskiy pereulok, Dolgoprudny, Moscow Region, 141700, \\ Russia \\ ${ }^{2}$ P.N. Lebedev Physical Institute of the Russian Academy of Sciences, 53 Leninskiy Prospekt, Moscow, \\ 119991, Russia
}

\begin{abstract}
A present status of experimentally observed states of excited charmed baryons is given. The classification of charmed baryons is presented, a quark model for ground states is briefly described, and the energy levels of excited states are analyzed.
\end{abstract}

The spectroscopy of charmed baryons is beautiful and intricate. With three quarks there are numerous degrees of freedom, giving rise to many more states than in the charmed meson sector. At the same time the large difference in mass between the charm quark and the light quarks provides a natural way to classify and understand these states: Heavy Quark Effective Theory (HQET). The spectrum of known singly-charmed states can be thought of in three broad regimes: the ground states, which are a vindication of the constituent quark model; the low-lying excited states, which are described well by HQET; and higher excited states, where the situation is murkier.

\section{Quark Model for Ground States}

In the constituent quark model [1], baryons composed of $u, d, s, c$ quarks can be classified into $S U(4)$ multiplets according to the symmetry of their flavor, spin, and spatial wavefunctions. All states in a given $S U(4)$ multiplet have the same angular momentum $J$, and parity $P$, but can have different quark flavors. For excited states with multiple units of orbital angular momentum the number of possible multiplets becomes large, but for the ground states the picture is much simpler. This framework is not exact - different states with the same conserved quantum numbers will mix, and baryons are not pure three-quark objects - but it works remarkably well for the ground states.

Quarks are fermions, so the baryon wavefunction must be overall antisymmetric under quark interchange ${ }^{1}$. Baryons are color singlets, and so have an antisymmetric color wavefunction. In the ground state, the orbital angular momentum $L$ is zero ( $S$-wave) and the spatial wavefunction is symmetric. Therefore, the product of the spin and flavor wavefunctions must also be symmetric for ground-state baryons. There are two ways this can be accomplished: both wavefunctions can be fully symmetric, or both can have mixed symmetry with the product being symmetric.

In concrete terms, we can consider a singly-charmed baryon to consist of a heavy $c$ quark and a light diquark with spin-parity $j^{p}$. Assuming isospin symmetry and letting $q$ denote a $u$ or $d$ quark, there are four possibilities for the flavor content of the diquark:

\footnotetext{
^e-mail: lena@lebedev.ru

${ }^{1}$ Strictly, it only needs to be antisymmetric under interchange of equal-mass quarks, but in order to build the model we assume $S U(4)$ is a good symmetry.
} 

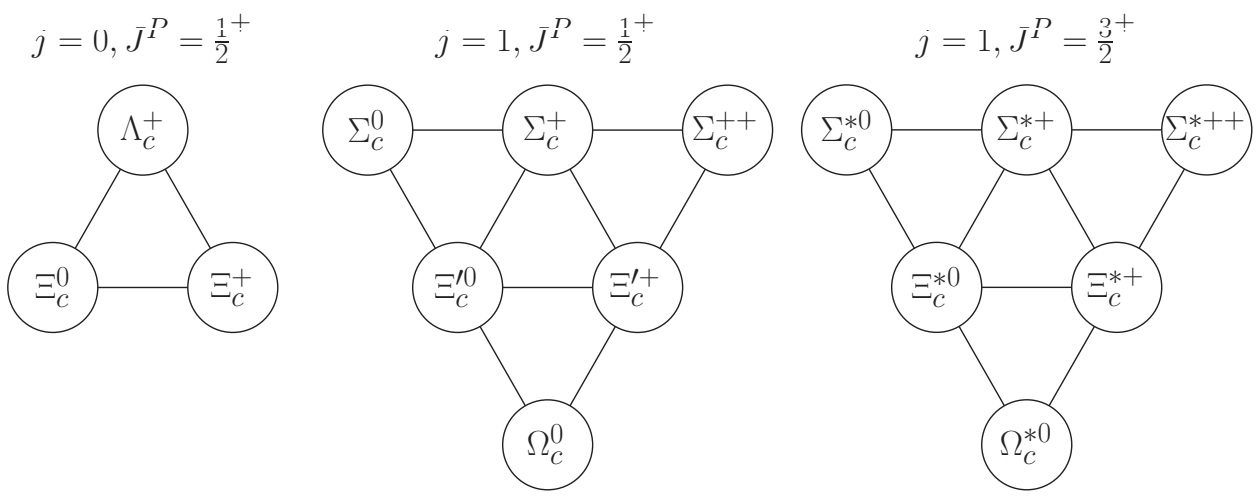

Figure 1. The $S U(3)$ multiplets containing the ground state baryons, grouped according to the spin $j$ of the light diquark and the spin-parity $J^{P}$ of the baryon.

- $q q$ with isospin 0 (flavor antisymmetric);

- $q q$ with isospin 1 (flavor symmetric);

- $s q$ with isospin $1 / 2$ (either);

- $s s$ with isospin 0 (flavor symmetric).

These correspond to the $\Lambda_{c}, \Sigma_{c}, \Xi_{c}$, and $\Omega_{c}$ states, respectively. The diquark wavefunction must be antisymmetric under quark interchange. Its color wavefunction is antisymmetric and in the ground state its spatial wavefunction is symmetric, so it may be either flavor-symmetric and spin-symmetric $\left(j^{p}=1^{+}\right)$or flavor-antisymmetric and spin-antisymmetric $\left(j^{p}=0^{+}\right)$. Combining the diquark with the charm quark gives rise to the possible states illustrated in figure 1 , where the multiplets of the full $S U(3)$ symmetry (formed by the $u, d$, and $s$ quarks) are shown. Those with $J^{P}=1 / 2^{+}$are all members of the same multiplet as the proton, and those with $J^{P}=3 / 2^{+}$are all members of the same multiplet as the $\Delta$ and $\Omega$. Note that there is a second isospin doublet of $\Xi_{c}$ states with $J^{P}=1 / 2^{+}$, denoted $\Xi_{c}^{\prime}$.

The constituent quark model predicts relations between the masses of these states as well as their existence and quantum numbers.

\section{Excited States}

Baryons can be given orbital $(l)$ or radial $(k)$ excitations. Since they are three-body systems there are two degrees of freedom in each case (denoted $\rho, \lambda$ ). For baryons with one heavy quark (mass $M$ ) and two light quarks (mass $m$ ), a natural way to specify these is to divide the system into a light diquark and the heavy quark. Taking a simple potential model based on the harmonic oscillator, the energy levels are given by ([2]):

$$
E=\sqrt{\frac{K}{m}}\left(3+2 l_{\rho}+4 k_{\rho}\right)+\sqrt{\frac{K}{\mu}}\left(3+2 l_{\lambda}+4 k_{\lambda}\right),
$$

where $K$ is a constant describing the potential and $\mu=(2 / 3 M+1 / 3 m)^{-1} \approx 3 m$ in the heavy quark limit. Thus, the $\rho$ excitations (within the diquark) require roughly three times as much energy as the 
Table 1. Summary of excited $\Lambda_{c}$ baryons [3].

\begin{tabular}{|c|c|c|c|c|}
\hline State & Decay mode & Mass, MeV/c & Width, MeV & $J^{P}$ \\
\hline \hline$\Lambda_{c}(2595)^{+}$ & $\Lambda_{c}^{+} \pi^{+} \pi^{-}, \Sigma_{c} \pi$ & $2592.3 \pm 0.3$ & $2.6 \pm 0.6$ & $\frac{1}{2}^{-}$ \\
\hline$\Lambda_{c}(2625)^{+}$ & $\Lambda_{c}^{+} \pi^{+} \pi^{-}, \Sigma_{c} \pi$ & $2628.11 \pm 0.19$ & $<0.97 @ 90 \% \mathrm{CL}$ & $\frac{3}{2}^{-}$ \\
\hline$\Lambda_{c}(2765)^{+}$ & $\Lambda_{c}^{+} \pi^{+} \pi^{-}, \Sigma_{c} \pi$ & $2766.6 \pm 2.4$ & $\sim 50$ & \\
\hline$\Lambda_{c}(2880)^{+}$ & $\Lambda_{c}^{+} \pi^{+} \pi^{-}, \Sigma_{c} \pi$, \\
& $\Sigma_{c}(2520) \pi, D^{0} p$ & $2881.5 \pm 0.4$ & $5.8 \pm 1.1$ & $\frac{5}{2}^{+}$ \\
\hline$\Lambda_{c}(2940)^{+}$ & $D^{0} p, \Sigma_{c} \pi$ & $2939.3_{-1.5}^{+1.4}$ & $17_{-6}^{+8}$ & \\
\hline
\end{tabular}

corresponding $\lambda$ excitations (between heavy quark and diquark). Therefore the lowest-lying excitations are those with $l_{\lambda}=1$ and the other quantum numbers zero, i.e. $L=1$. (Within this band there will be further splitting, e.g. due to spin-spin and spin-orbit couplings.) The second band will consist of two groups of states that have comparable energy: those with $l_{\lambda}=2(L=2)$ and those with $k_{\lambda}=1$ $(L=0)$, with the other quantum numbers being zero. Beyond the second band the degeneracy grows further, but we lack useful experimental data in this region in any case.

All this said, it is important to bear in mind that states which share all conserved, external quantum numbers $(J, P, I, C, S)$ can mix. Therefore we should be careful when interpreting observed resonances as specific expected states, particularly for higher excitations.

\section{$3 \Lambda_{c}$ Family}

Table 1 summarizes the excited $\Lambda_{c}^{+}$baryons. The first two lowest-lying states listed, namely the $\Lambda_{c}(2595)^{+}$and $\Lambda_{c}(2625)^{+}$, are not far above the $\Lambda_{c}^{+} \pi^{+} \pi^{-}$threshold and considered to be well established. The measured masses and decay patterns suggest that they are orbitally excited $\Lambda_{c}^{+}$ baryons with total angular momentum of the light quarks $L=1$. Thus their quantum numbers are assigned to be $J^{P}=\left(\frac{1}{2}\right)^{-}$and $J^{P}=\left(\frac{3}{2}\right)^{-}$, respectively. Their mass measurements are dominated by CDF [4]: $M\left(\Lambda_{c}(2595)^{+}\right)=(2592.25 \pm 0.24 \pm 0.14) \mathrm{MeV} / c^{2}$ and $M\left(\Lambda_{c}(2625)^{+}\right)=$ $(2628.11 \pm 0.13 \pm 0.14) \mathrm{MeV} / c^{2}$. Earlier measurements did not fully take into account the restricted phase space of the $\Lambda_{c}(2595)^{+}$decays.

The next two states, $\Lambda_{c}(2765)^{+}$and $\Lambda_{c}(2880)^{+}$, were discovered by CLEO [5] in the $\Lambda_{c}^{+} \pi^{+} \pi^{-}$final state. CLEO found that a significant fraction of the $\Lambda_{c}(2880)^{+}$decays proceeds via an intermediate $\Sigma_{c}(2445)^{++/ 0} \pi^{-/+}$. Later BaBar [6] observed that this state has also a $D^{0} p$ decay mode. This was the first example of an excited charmed baryon decaying into a charm meson plus a baryon; previously all excited charmed baryon were found in their hadronic transitions into lower lying charmed baryons. In the same analysis BaBar observed for the first time an additional state, $\Lambda_{c}(2940)^{+}$, decaying into $D^{0} p$. Studying the $D^{+} p$ final state, BaBar found no signal; this implies that the $\Lambda_{c}(2880)^{+}$and $\Lambda_{c}(2940)^{+}$ are $\Lambda_{c}^{+}$excited states rather than $\Sigma_{c}$ excitations. Belle reported the result of an angular analysis that favors $5 / 2$ for the $\Lambda_{c}(2880)^{+}$spin hypothesis. Moreover, the measured ratio of branching fractions $\mathcal{B}\left(\Lambda_{c}(2880)^{+} \rightarrow \Sigma_{c}(2520) \pi^{ \pm}\right) / \mathcal{B}\left(\Lambda_{c}(2880)^{+} \rightarrow \Sigma_{c}(2455) \pi^{ \pm}\right)=(0.225 \pm 0.062 \pm 0.025)$, combined with theoretical predictions based on HQS [7], favor even parity. However this prediction is only valid if the P-wave portion of $\Sigma_{c}(2520) \pi$ is suppressed.

The current open questions in the excited $\Lambda_{c}^{+}$family include the determination of quantum numbers for the other states, and the nature of the $\Lambda_{c}(2765)^{+}$state, in particular whether it is an excited $\Sigma_{c}^{+}$or $\Lambda_{c}^{+}$. However, there is no doubt that the state exists, as it is clearly visible in Belle data. 
Table 2. Summary of excited $\Sigma_{c}$ baryons [3].

\begin{tabular}{|l|c|c|c|c|}
\hline \multicolumn{1}{|c|}{ State } & Decay mode & Mass, MeV/c & Width, MeV & $J^{P}$ \\
\hline \hline$\Sigma_{c}(2520)^{++}$ & $\Lambda_{c}^{+} \pi^{+}$ & $231.95_{-0.12}^{+0.17}$ & $14.78_{-0.4}^{+0.3}$ & $\frac{3}{2}^{+}$ \\
$\Sigma_{c}(2520)^{+}$ & $\Lambda_{c}^{+} \pi^{0}$ & $231.0 \pm 2.3$ & $<17 @ 90 \% \mathrm{CL}$ & $\frac{3}{2}^{+}$ \\
$\Sigma_{c}(2520)^{0}$ & $\Lambda_{c}^{+} \pi^{-}$ & $232.02_{-0.14}^{+0.15}$ & $15.3_{-0.5}^{+0.4}$ & $\frac{3}{2}^{+}$ \\
\hline$\Sigma_{c}(2800)^{++}$ & $\Lambda_{c}^{+} \pi^{+}$ & $514_{-6}^{+4}$ & $75_{-17}^{+22}$ & $\frac{3}{2}^{-}$ \\
$\Sigma_{c}(2800)^{+}$ & $\Lambda_{c}^{+} \pi^{0}$ & $505_{-5}^{+14}$ & $62_{-40}^{+60}$ & $\frac{3}{2}^{-}$ \\
$\Sigma_{c}(2800)^{0}$ & $\Lambda_{c}^{+} \pi^{-}$ & $519_{-7}^{+5}$ & $72_{-15}^{+22}$ & $\frac{3}{2}^{-}$ \\
\hline
\end{tabular}

\section{$4 \Sigma_{c}$ Family}

Table 2 summarizes the excited $\Sigma_{c}^{++,+, 0}$ baryons. The first isotriplet of excited $\Sigma_{c}^{++,+, 0}$ baryons, $\Sigma_{c}(2520)^{++,+0}$, is well established. The only kinematically allowed strong decay for it is $\Sigma_{c} \rightarrow \Lambda_{c}^{+} \pi$. Belle [8] precisely measured the mass differences and widths of the doubly charged and neutral members of this triplet.

The short list of excited $\Sigma_{c}$ baryons is completed by the triplet of $\Sigma_{c}(2800)$ states observed by Belle [9]. Based on the measured masses and theoretical predictions [10], these states are assumed to be members of the predicted $\Sigma_{c 2} 3 / 2^{-}$triplet. From a study of resonant substructure in $B^{-} \rightarrow$ $\Lambda_{c}^{+} \bar{p} \pi^{-}$decays, BaBar found a significant signal in the $\Lambda_{c}^{+} \pi^{-}$final state with a mean value higher than measured by Belle for the $\Sigma_{c}(2800)$ by about $3 \sigma$ (Table 2 ). The decay widths measured by Belle and $\mathrm{BaBar}$ are consistent, but it is an open question if the observed state is the same as the Belle state.

\section{$5 \Xi_{c}$ Family}

Since all three quark flavors are different for the $\Xi_{c}$, there are many allowed configurations. These may be divided into states for which the light diquark wavefunction is flavor-antisymmetric (analogous to $\Lambda_{c}$ ) or flavor-symmetric (analogous to $\Sigma_{c}$ ).

The two ground states are the only members of the group that decay weakly, and their masses, lifetimes, and many of their decay modes have been measured [3]. The $\Xi_{c}$ states also exist in many angular momentum configurations of the constituent quarks, each as an isospin pair (Table 3).

The $\Xi_{c}^{\prime}$ and $\Xi_{c}(2645)$ states form a doublet analogous to the $\Sigma_{c}(2455)$ and $\Sigma_{c}(2520)$ with expected $\left(j^{p}, J^{P}\right)$ of $\left(1^{+}, 1 / 2^{+}\right)$and $\left(1^{+}, 3 / 2^{+}\right)$, respectively. The former is too light to decay strongly, but the electromagnetic transition $\Xi_{c}^{\prime} \rightarrow \Xi_{c} \gamma$ is allowed. BaBar performed an angular analysis of $\Xi_{c}^{\prime 0} \rightarrow \Xi_{c}^{0}(\rightarrow$ $\left.\Xi^{-} \pi^{+}\right) \gamma$ in the helicity formalism and found the data to be consistent with $J=1 / 2$ [11]. However, due to the inclusive production environment higher spins could not be ruled out.

The low-lying excited states $\Xi_{c}(2790)$ and $\Xi_{c}(2815)$ are analogous to the $\Lambda_{c}(2595)$ and $\Lambda_{c}(2625)$, and their decays follow a corresponding pattern: $\Xi_{c}(2790) \rightarrow \Xi_{c}^{\prime} \pi, \Xi_{c}(2815) \rightarrow \Xi_{c}(2645) \pi$. They were therefore identified as the $1 / 2^{-}, 3 / 2^{-}$doublet with $j^{p}=1^{-}$and the diquark in a flavorantisymmetric configuration [12]. Recently Belle collaboration significantly improved measured mass differences (between excited and ground states) and widths (where applicable) for $\Xi_{c}^{\prime}, \Xi_{c}(2645)$, $\Xi_{c}(2790), \Xi_{c}(2815)$, and $\Xi_{c}(2970)$ states [13].

The $\Xi_{c}$ (2930) was seen in $B^{-} \rightarrow \Lambda_{c}^{+} \Lambda_{c}^{-} K^{-}$decays [14]. The Dalitz plot was clearly not flat and the $\Lambda_{c}^{+} K^{-}$projection was consistent with a single resonance with the parameters given in Table 3 . However, given the small sample size and the inability to rule out other explanations (such as two overlapping $\Xi_{c}$ resonances or a complicated interference pattern between $\Xi_{c}$ and charmonium resonances) this is considered unconfirmed. 
Table 3. Summary of excited $\Xi_{c}$ baryons [3].

\begin{tabular}{|c|c|c|c|c|}
\hline State & Decay mode & Mass, MeV/c & Width, MeV & $J^{P}$ \\
\hline \hline$\Xi_{c}^{\prime+}$ & $\Xi_{c}^{+} \gamma$ & $2577.4 \pm 1.2$ & & $\frac{1}{2}^{+}$ \\
$\Xi_{c}^{\prime 0}$ & $\Xi_{c}^{0} \gamma$ & $2578.8 \pm 0.5$ & & $\frac{1}{2}^{+}$ \\
\hline$\Xi_{c}(2645)^{+}$ & $\Xi_{c}^{0} \pi^{+}$ & $2645.53 \pm 0.31$ & $2.14 \pm 0.19$ & $\frac{3}{2}^{+}$ \\
$\Xi_{c}(2645)^{0}$ & $\Xi_{c}^{+} \pi^{-}$ & $2646.32 \pm 0.31$ & $2.35 \pm 0.22$ & $\frac{3}{2}^{+}$ \\
\hline$\Xi_{c}(2790)^{+}$ & $\Xi_{c}^{\prime 0} \pi^{+}$ & $2792.0 \pm 0.5$ & $8.9 \pm 1.0$ & $\frac{1}{2}^{-}$ \\
$\Xi_{c}(2790)^{0}$ & $\Xi_{c}^{\prime+} \pi^{-}$ & $2792.8 \pm 1.2$ & $10.0 \pm 1.1$ & $\frac{1}{2}^{-}$ \\
\hline$\Xi_{c}(2815)^{+}$ & $\Xi_{c}^{+} \pi^{+} \pi^{-}, \Xi_{c}(2645)^{0} \pi^{+}, \Xi_{c}^{0 \prime} \pi^{+}$ & $2816.67 \pm 0.31$ & $2.43 \pm 0.26$ & $\frac{3}{2}^{-}$ \\
$\Xi_{c}(2815)^{0}$ & $\Xi_{c}^{0} \pi^{+} \pi^{-}, \Xi_{c}(2645)^{+} \pi^{-}, \Xi_{c}^{+\prime} \pi^{-}$ & $2820.22 \pm 0.32$ & $2.54 \pm 0.25$ & $\frac{3}{2}^{-}$ \\
\hline$\Xi_{c}(2930)^{0}$ & $\Lambda_{c}^{+} K^{-}$ & $2931 \pm 6$ & $36 \pm 13$ & \\
\hline$\Xi_{c}(2970)^{+}$ & $\Lambda_{c}^{+} K^{-} \pi^{+}, \Sigma_{c}^{++} K^{-}, \Xi_{c}(2645)^{0} \pi^{+}, \Xi_{c}^{0 \prime} \pi^{+}$ & $2969.4 \pm 0.8$ & $20.9_{-3.5}^{+2.4}$ & \\
$\Xi_{c}(2970)^{0}$ & $\Xi_{c}(2645)^{+} \pi^{-}, \Xi_{c}^{++} \pi^{-}$ & $2967.8 \pm 0.8$ & $28.1_{-4.0}^{+3.4}$ & \\
\hline$\Xi_{c}(3055)^{+}$ & $\Sigma_{c}^{++} K^{-}, \Lambda D^{+}$ & $3055.9 \pm 0.4$ & $7.8 \pm 1.9$ & \\
$\Xi_{c}(3055)^{0}$ & $\Lambda D^{0}$ & $3059.0 \pm 0.8$ & $6.4 \pm 2.4$ & \\
\hline$\Xi_{c}(3080)^{+}$ & $\Lambda_{c}^{+} K^{-} \pi^{+}, \Sigma_{c}^{++} K^{-}, \Sigma_{c}(2520)^{++} K^{-}, \Lambda D^{+}$ & $3077.2 \pm 0.4$ & $3.6 \pm 1.1$ & \\
$\Xi_{c}(3080)^{0}$ & $\Lambda_{c}^{+} K_{S}^{0} \pi^{-}, \Sigma_{c}^{0} K_{S}^{0}, \Sigma_{c}(2520)^{0} K_{S}^{0}$ & $3079.9 \pm 1.4$ & $5.6 \pm 2.2$ & \\
\hline \multicolumn{4}{|c|}{}
\end{tabular}

The remaining resonances were all seen in the $\Lambda_{c}^{+} \bar{K} \pi^{+}$isodoublet of final states (and, in the case of the $\Xi_{c}(2970)$, in $\left.\Xi_{c}(2645) \pi\right)$. The $\Xi_{c}(2970)$ and $\Xi_{c}(3077)$ were discovered by Belle in $\Lambda_{c}^{+} \bar{K} \pi$ and confirmed by BaBar. Since this is a three-body decay it could proceed via an intermediate $\Sigma_{c}$. BaBar tested this by fitting a two-dimensional PDF in $M\left(\Lambda_{c}^{+} \pi\right), M\left(\Lambda_{c}^{+} \bar{K} \pi\right)$. It was found that approximately half of the $\Xi_{c}(2970)$ decays to this final state proceed through an intermediate $\Sigma_{c}(2455)$ with the rest non-resonant. By contrast, most if not all of the $\Xi_{c}(3077)$ decays to this final state proceed via $\Sigma_{c}(2455)$ or $\Sigma_{c}(2520)$ with approximately equal branching fractions to each. Because the $\Xi_{c}(2970)$ is close to threshold on the scale of its natural width, especially with an intermediate $\Sigma_{c}$, the available phase space changes significantly across the resonance. Different handling of this threshold behavior is the reason for the mild tension in the fitted $\Xi_{c}(2970)$ masses between [15] and [16]. The masses measured in the $\Xi_{c}(2645) \pi^{+}$final state [17], which is far from threshold, are consistent with the BaBar treatment, although the widths are smaller than either experiment saw in $\Lambda_{c}^{+} \bar{K} \pi$. Requiring an intermediate $\Sigma_{c}$ reduces the background levels, and by doing this BaBar was able to identify two further candidate states, the $\Xi_{c}(3055)$ and $\Xi_{c}(3123)$. The latter had a limited statistical significance $(3 \sigma)$, and later was not confirmed by Belle with twice as much statistics [18].

Till recently all measurements of excited $\Xi_{c}$ baryons were performed using decay modes in which the charm quark is contained in the final state baryon. Belle reported studies of $\Xi_{c}$ baryons decaying to the $\Lambda D^{+}$and $\Lambda D^{0}$ final states [19]. Significant signals were found for $\Xi_{c}(3055)^{+}$and $\Xi_{c}(3080)^{+}$ decays into $\Lambda D^{+}$. In the $\Lambda D^{0}$ final state observation of the $\Xi_{c}(3055)^{0}$ was reported.

As a final point it should be noted that no direct measurements of the $J^{P}$ of any of the excited $\Xi_{c}$ states are available. Mild constraints on the quantum numbers can be inferred from the decay pattern. For example, the observation of the $\Xi_{c}(3077)$ in $\Sigma_{c} \bar{K}$ and $\Sigma_{c}(2520) \bar{K}$ excludes states with diquark $j^{p}=0^{-}$. However, many quantum numbers are still allowed for these states and there is a range of opinions on the best match to the data. 
Table 4. Summary table of excited $\Omega_{c}$ baryons. For each parameter, the first uncertainty is statistical and the second systematic. The asymmetric uncertainty arising from the $\Xi_{c}^{+}$mass is given separately.

\begin{tabular}{|c|c|c|c|c|}
\hline State & Decay mode & Mass, MeV/c & Width, MeV & $J^{P}$ \\
\hline \hline$\Omega_{c}^{* 0}$ & $\Omega_{c}^{0} \gamma$ & $2765.9 \pm 2.0$ & & $\frac{3}{2}^{+}$ \\
\hline$\Omega_{c}(3000)^{0}$ & & $3000.4 \pm 0.2 \pm 0.1_{-0.5}^{+0.3}$ & $4.5 \pm 0.6 \pm 0.3$ & \\
$\Omega_{c}(3050)^{0}$ & & $3050.2 \pm 0.1 \pm 0.1_{-0.5}^{+0.3}$ & $0.8 \pm 0.2 \pm 0.1$ & \\
$\Omega_{c}(3066)^{0}$ & $\Xi_{c}^{+} K^{-}$ & $3065.6 \pm 0.1 \pm 0.3_{-0.5}^{+0.3}$ & $3.5 \pm 0.4 \pm 0.2$ & \\
$\Omega_{c}(3090)^{0}$ & & $3090.2 \pm 0.3 \pm 0.5_{-0.5}^{+0.3}$ & $8.7 \pm 1.0 \pm 0.8$ & \\
$\Omega_{c}(3119)^{0}$ & & $3119.1 \pm 0.3 \pm 0.9_{-0.5}^{+0.3}$ & $1.1 \pm 0.8 \pm 0.4$ & \\
\hline
\end{tabular}

\section{$6 \Omega_{c}$ Family}

In contrast to the $\Lambda_{c}, \Sigma_{c}$, and $\Xi_{c}$ families available experimental data on the $\Omega_{c}$ ground states were limited before the $B$ Factories. The weakly-decaying $\Omega_{c}^{0}\left(J=1 / 2^{+}\right)$had been seen in a number of different decay modes and production environments, but with only limited statistics (typically samples of $O(10)$ events in a given decay mode, and never more than 100); and the $J=3 / 2^{+} \Omega_{c}^{* 0}$ had not been observed. Belle carried out a precise measurement of the $\Omega_{c}^{0}$ mass using a sample of 725 decays to the $\Omega^{-} \pi^{+}$final state [20], obtaining $\left(2693.6 \pm 0.3_{-1.5}^{+1.8}\right) \mathrm{MeV} / c^{2}$.

The short list of known doubly strange charmed baryons is summarized in Table 4 . The $\Omega_{c}^{* 0}$ is too light to undergo strong decay and so decays purely to $\Omega_{c}^{0} \gamma$. It was discovered by BaBar [21] and confirmed by Belle [20]. Both measured the mass difference $M\left(\Omega_{c}^{* 0}\right)-M\left(\Omega_{c}^{0}\right)$, and the two results are in excellent agreement with each other as well as with most theoretical predictions [22].

Recently LHCb Collaboration performed a study of the $\Xi_{c}^{+} K^{-}$spectrum, observing five new narrow excited $\Omega_{c}^{0}$ states [23] and thus presenting first excited doubly strange charmed baryons, that decay strongly. The observation of these states in an inclusive process through a two-body decay does not allow the determination of their quantum numbers. More information can be obtained from the study of possible three-body decays or when reconstructing these states in decays of heavy baryons.

\section{Summary}

Recently observed excited $\Omega_{c}$ states present a unique opportunity to test and further improve theoretical models, that predict properties of heavy hadrons. More accurate $\Xi_{c}$ mass values is of both practical and theoretical interest, and knowing their widths (where applicable) can then lead to measurements of the matrix elements of their decays. These matrix elements are also applicable to other excited charmed and bottom baryons.

Interesting feature of the charmed baryons spectrum is that highly excited charmed baryons can decay to a charmed meson and a non-charmed baryon.

It should be noted that no direct measurements of the $J^{P}$ of any of the excited strange charmed baryons are available. Constraints on the quantum numbers can be inferred only from the decay pattern.

\section{Acknowledgements}

This work was supported by the Russian Foundation for Basic Research grant No. 16-32-60134. 


\section{References}

[1] M. Gell-Mann, Phys. Lett. 8, 214 (1964);

G. Zweig, CERN-TH-401 (1964);

G. Zweig, Developments in the Quark Theory of Hadrons 1, 22 (1980).

[2] E. Klempt and J.-M. Richard, Rev. Mod. Phys. 82, 1095 (2010).

[3] C. Patrignani et al. (Particle Data Group), Chin. Phys. C 40, 100001 (2016), and 2017 update.

[4] T. Aaltonen et al. (CDF Collaboration), Phys. Rev. D 84, 012003 (2011).

[5] M. Artuso et al. (CLEO Collaboration), Phys. Rev. Lett. 86, 4479 (2001).

[6] B. Aubert et al. (BaBar Collaboration), Phys. Rev. Lett. 98, 012001 (2007).

[7] N. Isgur and M. B. Wise, Phys. Rev. Lett. 66, 1130 (1991),

H.-Y. Cheng and C.-K. Chua, Phys. Rev. D 75, 014006(2007).

[8] S. H. Lee et al. (Belle Collaboration), Phys. Rev. D 89, 091102 (2014).

[9] R. Mizuk et al. (Belle Collaboration), Phys. Rev. Lett. 94, 122002 (2005).

[10] L. Copley, N. Isgur, and G. Karl, Phys. Rev. D 20, 768 (1979), Erratum ibid. 23, 817 (1981),

D. Pirjol and T.-M. Yan, Phys. Rev. D 56, 5483 (1997).

[11] B. Aubert et al. (BaBar Collaboration), hep-ex/0607086 (2006).

[12] C. E. Csorna et al. (CLEO Collaboration), Phys. Rev. Lett. 86, 4243 (2001);

J. P. Alexander et al. (CLEO Collaboration), Phys. Rev. Lett. 83, 3390 (1999).

[13] J. Yelton et al. (Belle Collaboration), Phys. Rev. D 94, 052011 (2016).

[14] B. Aubert et al. (BaBar Collaboration), Phys. Rev. D 77, 031101 (2008).

[15] R. Chistov et al. (Belle Collaboration), Phys. Rev. Lett. 97, 162001 (2006).

[16] B. Aubert et al. (BaBar Collaboration), Phys. Rev. D 77, 012002 (2008).

[17] T. Lesiak et al. (Belle Collaboration), Phys. Lett. B 665, 9 (2008).

[18] Y. Kato, T. Iijima et al. (Belle Collaboration), Phys. Rev. D 89, 052003 (2014).

[19] Y. Kato, T. Iijima et al. (Belle Collaboration), Phys. Rev. D 94, 032002 (2016).

[20] E. Solovieva, R. Chistov et al. (Belle Collaboration), Phys. Lett. B 672, 1 (2009).

[21] B. Aubert et al. (BaBar Collaboration), Phys. Rev. Lett. 97, 232001 (2006).

[22] R. Roncaglia, B. Lichtenberg, and E. Predazzi, Phys. Rev. D 52, 1722 (1995);

L. Rosner, Phys. Rev. D 52, 6461 (1995);

J. Savage, Phys. Lett. B 359, 189 (1995);

B. Lichtenberg, R. Roncaglia, and E. Predazzi, Phys. Rev. D 53, 6678 (1996);

E. Jenkins, Phys. Rev. D 54, 4515 (1996);

Y. Glozman and D. Riska, Nucl. Phys. A 603, 326 (1996);

A. Zalewska and K. Zalewski, hep-ph/9608240 (1996);

L. Burakovsky, T. Goldman, and L. Horwitz, Phys. Rev. D 56, 7124 (1997);

N. Mathur, R. Lewis, and M. Woloshyn, Phys. Rev. D 66, 014502 (2002).

[23] R. Aaij et al. (LHCb Collaboration), Phys. Rev. Lett. 118, 182001 (2017). 